\title{
UNA AGENDA PARA LA INTELECTUALIDAD DE AMÉRICA LATINA Y EL CARIBE: ACOGIENDO LA HERENCIA DE LEOPOLDO ZEA PARA PENSAR MÁS ALLÁ DEL ESTADO-NACIÓN
}

\author{
Eduardo Devés-Valdés $\left.{ }^{1}{ }^{*}\right)$
}

\section{RESUMEN}

Ante la pregunta por las maneras en que la intelectualidad de América Latina y el Caribe (ALC) ha intentado pensar más allá del estado nación y frente a la inquietud surgida por una agenda metanacional pensada por-para esta misma intelectualidad se estudia la obra de Leopoldo Zea.

Se presentan cuatro niveles en los cuales Leopoldo Zea formuló una agenda metanacional para esta intelectualidad: los estudios eidéticos, el pensamiento y la filosofía de ALC, la creación de redes de estudios de ALC y la difusión mundial de la cultura mexicana. Esto se hace por relación a numerosas formulaciones realizadas por personas que intentaron evaluar la obra y la trayectoria del importante pensador mexicano: Werner Altmann, Carolina Guerrero, Abelardo Villegas, Enrique Dussel, Tzvi Medin, Javier Pinedo, Alberto Saladino, Fernando Aínsa, Pablo Guadarrama, entre otras.

Se intenta finalmente proyectar algunas ideas de Leopoldo Zea más allá de lo que él mismo formuló.

$$
\text { Palabras clave: }
$$

Pensamiento latinoamericano - pensamiento periférico - historia intelectual sociedad civil intelectual - paradigma nacional.

${ }^{*}$ ) Post-Doctorado, Universidad de Lovaina. Investigador del Instituto de Estudios Avanzados (IDEA) de la Universidad de Santiago de Chile.

Artículo recibido el 5 de abril de 2010. Aceptado por el Comité Editorial el 29 de septiembre de 2010.

Correo electrónico: eduardo.deves@usach.cl

${ }^{1}$ Román Díaz 89, Providencia, Santiago, Chile. Tel 56-2-7181360. 


\begin{abstract}
Facing the question about the forms in which the Latin American-Caribbean intellectuality has though beyond the nation-state and facing the question about a meta-national "agenda" entertained by this same intellectuality, I study the work of Leopoldo Zea.

I present four levels in which Leopoldo Zea built a meta-national "agenda" for the intellectuality above mentioned: the firs level related to eidetical studies, the second is about LAC philosophy and thought; the third level aims at the creation of intellectualnetworks in LAC; the last one refers to the spread out of Mexican culture worldwide. This article has been elaborated taking, also, a lot of contributions offered by authors who have written about this Mexican philosopher: Werner Altman, Carolina Guerrero, Abelardo Villegas, Enrique Dussel, Tzvi Medin, Javier Pinedo, Alberto Saladino, Fernando Aínsa, Pablo Guadarrama, among others.

Finally, I intend to launch some ideas of Leopoldo Zea beyond his own conceptions.
\end{abstract}

\title{
Keywords:
}

Latin-American thought - peripheral thought - intellectual history - intellectual civil society - national paradigm.

\section{INTRODUCCIÓN}

Proponer una agenda para quienes trabajan con el conocimiento es una tarea con dos caras: formular una manera de pensar y una manera de actuar para este grupo. Por cierto, ambas son dos dimensiones de una misma tarea.

Partiendo de la siguiente constatación: si América Latina y el Caribe (ALC) se encuentran en un estado claramente inferior al deseado y al proyectado, menoscabada respecto a otras regiones del mundo, con indicadores de crecimiento bajísimos en algunos rubros y negativos en otros, debe asumirse que hemos pensado mal y/o actuado mal. Los objetivos fueron inadecuados o lo fueron los medios para lograrlos, o ambas cosas. Lo más probable es que se trate de las dos y de otras más (ver Devés, 2007a).

Ahora bien, el objetivo de este trabajo es pensar una agenda para quienes trabajan con el conocimiento en ALC, teniendo como punto de partida (o como pretexto) uno de los más importantes intelectuales latinoamericanos, quien fue capaz de pensar a ALC como conjunto, imaginar escenarios de futuro y hacer propuestas para que la intelectualidad asumiera su papel: Leopoldo Zea.

Respecto de las ideas, Leopoldo Zea fue uno de los pocos intelectuales latinoamericanos sensibles a pensar con (o en) relación a otras regiones e inspirándose en otras 
regiones periféricas. No sólo citó en su obra a personas como K. Nkrumah², Leopold Senghor, Ahmed Sukarno, José Rizal entre otras, sino que a la vez se interesó por los contactos "Sur-Sur". Leopoldo Zea intentó pensar a ALC como parte del mundo periférico, comprendiendo allí a África y Asia, como también lo eslavo y lo ibérico. Su herencia (y su mandato) es muy clara en este sentido: hay que pensar al estado-nación más allá de sí mismo y a ALC más allá de ALC. Nuestro pensamiento debe abrirse, ampliarse y esponjarse, y uno de los mayores desafíos es ir más allá de sí mismo, superando un provincianismo estrecho, que es producto de la ignorancia y sobre todo de la pereza: para estudiar lo que se ha dicho en otros países, en otros continentes y en otras lenguas.

Una de sus principales destrezas consistió en la capacidad para elaborar redes intelectuales en el marco de ALC, aunque también más allá. Los ejemplos más importantes fueron la Sociedad Latinoamericana de Estudios sobre América Latina y el Caribe (SOLAR) y la Federación Internacional de Estudios sobre América Latina y El Caribe (FIEALC), pero no son únicos. Debe tenerse en cuenta que igualmente legó esta herencia a varias personas quienes han continuado su tarea. Aunque, sin duda Leopoldo Zea no fue el único que avanzó en la creación de redes en los espacios latinoamericano y periférico. Por señalar algunos casos igualmente relevantes, de la década de 1970, pueden ponerse en relieve las figuras de Samir Amin, Enrique Iglesias, Pablo González-Casanova, Mahbub ul-Haq, Dudley Seers, entre quienes, desde ALC o desde otros lugares, intentaron articularnos al resto del mundo intelectual.

\section{¿QUÉ ES ESTO DE ELABORAR UNA AGENDA PARA LA INTELECTUALIDAD Y LA SOCIEDAD?}

Numerosas intelectualidades, particularmente durante los últimos dos siglos, se han ocupado de formular agendas para sus naciones, culturas, sociedades o regiones: los indios en la sociedad Brahmo-Samaj hacia 1830, los japoneses de la sociedad Meirokusha hacia 1870, los panafricanistas en 1945 luego del Congreso de Manchester, los eslavófilos rusos hacia 1840, los nacionalistas-republicanos chinos durante la segunda década del XX, la corriente salafita, de al-Manar, dentro del islamismo hacia 1900, la generación romántica en A.L. hacia 1840 y la red del Repertorio Americano hacia 1925 y la generación del 1898 en España, son algunos de los casos más conocidos, entre muchos otros. Más recientemente, el filósofo africano Kwasi Wiredu se ha formulado la pregunta por una agenda para un continente que, como dice, no hay que ser muy astuto para darse cuenta de cuantas deficiencias posee. Wiredu formula una serie de tareas para esa urgente nueva filosofía en África, que tiene que apuntar en un sentido crítico, por un lado y reconstructivo, por otro, y que se relaciona con la

\footnotetext{
${ }^{2}$ Asumiendo la necesidad de una producción industrial de artículos, se ha optado por la opción que consiste en subir a Internet una gran cantidad de piezas, llamémoslas "accesorias", de modo de no tener que incorporarlas a cada artículo, especialmente si van a ser publicados en papel. Ello implicaría un encarecimiento sustancial, por el crecimiento del texto. Así, una gran cantidad de personas, escuelas de pensamiento, instituciones y redes, pueden consultarse en ATLAS DEL PENSAMIENTO en www.umbral.uprrp.edu. Allí podrán encontrarse miles de otras informaciones y miles más de conexiones, para quienes deseen profundizar en estos temas. El proyecto ATLAS DEL PENSAMIENTO, como su nombre lo indica, apunta a exhibir geográficamente el pensamiento, particularmente de las regiones periféricas, durante los 3 últimos siglos. En el texto impreso se entregan únicamente las referencias más necesarias.
} 
descolonización, con el desarrollo de los hábitos mentales característicos de la ciencia, con la necesidad de repensar los fundamentos con el aprovechamiento del acervo intelectual y cultural de la región (1995, 4ss, 13ss y 20). Sin duda, las propuestas del africano deben ser al menos parcialmente distintas a las de ALC pero interesa poner en relieve su intención.

Leopoldo Zea no estuvo al margen de esta intención agendaria. Existen numerosos textos suyos en que va enunciando las propuestas directivas para una acción: algunas insertas en el marco de investigaciones y trabajos sobre historia de las ideas, otras explícitamente en artículos, discursos y presentaciones en reuniones o congresos. En múltiples oportunidades aprovechó a figuras históricas u ocasiones -el Ateneo mexicano, el noventaiochismo español, a Simón Bolívar, a José Vasconcelos o a José Martí- para insinuar sus propuestas respecto a la tarea intelectual en ALC. La pregunta por la agenda que formula Leopoldo Zea para sí mismo, como epónimo de la intelectualidad latinoamericana, tiene varios niveles: la conciencia (autoconciencia) de México, la posibilidad y necesidad de filosofar a la altura del hombre, la posibilidad u obligación para ALC de hacer un aporte a la cultura mundial. En este caso, nos interesa la intersección entre agenda y capacidad para pensar más allá del estado-nación. En consecuencia, existen varios elementos de la agenda formulada por Leopoldo Zea que para esta pregunta carecen de relevancia, otras en cambio, adquieren una relevancia inusitada: la tarea de una autoconciencia latinoamericana, la tarea del aporte a la cultura mundial, la tarea de constituir una red de estudios de ALC. Por otra parte, debe distinguirse nítidamente entre la agenda para la intelectualidad y para la sociedad como conjunto, aunque haya múltiples traslapes entre ambos niveles.

Desde temprano, y en el marco de preocupaciones de cara a la intelectualidad europea existencialista de la segunda post-guerra, en El peligro de la libertad intelectual de1950, argumenta que
“el intelectual tiene una misión social; él como todos los hombres, está comprometido con su sociedad y es responsable ante ella. No es ni puede ser en modo alguno, un individuo con prerrogativas especiales. Su inteligencia no es fuero y por lo mismo, tampoco es excusa de responsabilidad. De los riesgos que corra la sociedad, el intelectual es tanto más responsable que el resto de los individuos que la forman. Pero esta responsabilidad es algo que tiene que ser asumido libremente, esto es, conscientemente. No son las dictaduras las encargadas de señalar a los intelectuales la misión que les corresponde, de acuerdo con los intereses perseguidos por las mismas, son los intelectuales mismos los que tienen que tomar conciencia de su papel social y realizarlo. Es en este acto donde la libertad intelectual se hace plenamente patente".

Ya no se habla de la libertad por la libertad, sino de una libertad responsable, esto es, de una libertad comprometida con las mejores y más altas aspiraciones del hombre (1959, pp. 106-108). Años más tarde, formula la noción: "A lo universal por lo profundo" en que, por así decirlo, el estado-nación aparece como trampolín para saltar hacia el mundo. "A lo universal por lo profundo" apunta la actitud de inteligencia mexicana, expresada a lo largo de la historia de nuestra cultura y en forma extraordinaria, afirma Leopoldo Zea, en los tiempos modernos, por José Vasconcelos, Alfonso Reyes 
y Octavio Paz. Lo profundo, lugar donde se dan cita las diversas expresiones de lo humano, multirraciales y multiculturales. "Somos por primera vez dice Octavio Paz contemporáneos de todos los hombres". Lo profundo, como base nuestra, es el llamado mundo indígena, recipiente de otras razas y culturas: fue con su mezcla que se integró y creó lo que Vasconcelos llama "raza cósmica" (1999, p. 27).

Pero la lucha por la libertad o la búsqueda de lo profundo no son necesariamente agendas que se piensen para ir más allá del estado-nación, ese esfuerzo por pensar más allá del estado-nación que es palpable en la obra de Leopoldo Zea, en diversas formas, a través de su larga carrera académica de más de 60 años. Por otra parte, sus viajes de trabajo por ALC y todo el mundo, sus estudios sobre nuestro pensamiento, y en las conexiones o paralelos entre éste y el de Asia y África, sus tareas de organizador de redes de estudios sobre ALC y su papel como difusor de la cultura mexicana en el mundo son todas formas de proyectarse más allá del estado-nación.

Ahora bien, el objetivo de este trabajo es mostrar las maneras en que este personaje relevantísimo del quehacer latinoamericano e importante gestor cultural de la región fue capaz de pensar más allá del estado-nación y qué posibilidades ofrece su ejemplo para mejorar la capacidad de superar un pensamiento circunscrito al estado-nación, que es una de las limitaciones que inhiben a la intelectualidad latinoamericana todavía en la actualidad. Las limitantes de pensar sólo nacionalmente inhiben la capacidad de integración, la capacidad de sinergizar las actividades intelectuales y muchas otras que se verían potenciadas si la intelectualidad de ALC pudiera pensar (y actuar, por cierto) más allá del estado-nación.

Pero ¿en qué sentido este pensamiento aldeanista inhibe el quehacer de la intelectualidad de ALC?

\section{UNA AGENDA PARA LOS ESTUDIOS EIDÉTICOS}

Los estudios eidéticos son, como se ha visto, una de las preocupaciones más relevantes y tematizadas de Leopoldo Zea. A través de los años, desde mediados de los cuarenta hasta el fin de su carrera, va formulando criterios para orientar el quehacer eidológico y, en muchas oportunidades, uno que debe trascender al estado-nación.

Como es muy normal, el trabajo de Leopoldo Zea en tanto que estudioso de las ideas, se inició con un desafío nacional referido al positivismo en México. Muy pronto, sin embargo, transitó hacia estudios con una dimensión regional, con Dos etapas en el pensamiento latinoamericano: del romanticismo al positivismo. Después de publicar El Positivismo en México, escribió; "desde entonces mis estudios se han conducido a buscar relaciones con experiencias semejantes de otros pueblos (...) y la búsqueda de una interpretación de nuestra historia, la historia de esos pueblos y el nuestro y la relación que esta historia guarda con la historia universal" (1968, p. 13). Constata que aquello que había iniciado como búsqueda de identidad para el caso mexicano no era privativo de éste. La conciencia en el propio Leopoldo Zea de un proyecto ampliado, se estaba realizando en otros lugares de ALC. Allí estaban Martínez Estrada, Borges, 
Mallea o Murena en Argentina, pero a la vez en un lugar geográficamente más cercano pero culturalmente más lejano, el Caribe de colonización francesa, A. Césaire y F. Fanon estaban embarcados en similar empresa y, más lejos todavía, en África, Leopold Senghor hacía, por su parte, lo mismo (1978b, XI). Mas allá de esto, Leopoldo Zea entró en diversos autores de las regiones periféricas, con el fin de establecer relaciones o paralelos entre las ideas de éstos y lo que ocurría en ALC (ver Devés 2007c, Devés y Melgar-Bao 2005, Gorski 1994).

Tres son los criterios que pueden desprenderse de las propuestas de Leopoldo Zea:

1) El estudio de las ideas debe mostrar lo común de la región, "conciencia sobre sí misma, argumenta en 1963, que origina una especie de nacionalismo cultural y filosófico, que da sentido a una de las mayores expresiones del pensamiento latinoamericano contemporáneo" (1976a, p. 13);

2) El estudio de las ideas debe contribuir a la conciencia latinoamericana, "a la búsqueda de la identidad del hombre de esta América, a partir de la cual podrá exigir el reconocimiento de su humanidad" (1976a, p. 9);

3) El estudio de las ideas debe contribuir a la emancipación o liberación de la región, "el título de este libro Discurso desde la marginación y la barbarie, hace referencia al discurso desde otras expresiones de hombres que no por ser distintas son menos humanas. La marginación y la barbarie como nuevos e ineludibles centros de expresión del hombre que, de esta forma, niegan la misma marginación y la barbarie" (1990, p. 19).

Si puede formularse más sintéticamente, los estudios eidéticos superando lo nacional y mostrando lo común de las ideas de la región, deben contribuir a la conciencia regional y por ese camino contribuir a la liberación de la propia región.

\section{UNA AGENDA PARA EL PENSAMIENTO Y LA FILOSOFÍA DE ALC}

En el siglo XX el pensamiento político latinoamericano se empeña, afirma en 1974, en la búsqueda de soluciones que pongan fin a la dependencia, el subdesarrollo y el neocolonialismo, y el pensamiento filosófico enfrenta el problema de la enajenación de la conciencia que han venido sufriendo los pueblos latinoamericanos, a través de las diversas etapas de su historia, para ponerle fin (1976a, pp. 9-10). En un texto más tardío Leopoldo Zea vuelve sobre algunos de estos asuntos. Respondiendo a una pregunta del filósofo uruguayo Fernando Aínsa, Leopoldo Zea argumenta que:

\footnotetext{
nuestros pueblos tienen enormes problemas por resolver, problemas de identidad, de dependencia. Por lo tanto la filosofía es un instrumento extraordinario para enfrentarlos y para tratar de darles solución. Una responsabilidad de los filósofos de la región es traducir y adaptar a la propia realidad lo que puede servir de otra (Entrevista con Aínsa 1993, pp. 24-25).
}

Pero para que ello sea posible es necesaria "una filosofía de la historia que haga posible la aufhebung, la absorción del pasado, la historia, una realidad que quiera o 
no, es la propia de esta América. Un pasado hecho de yuxtaposiciones que, al hacerse consciente, se presenta como algo propio y del cual, por serlo, hay que partir para construir el presente y el futuro más propios. Una filosofía de la historia que ponga fin a la situación, trágicamente expresada por el libertador Simón Bolívar cuando decía 'Hemos arado en el mar'. Eso es, a la idea de que, una y otra vez, se ha venido laborando sobre el vacío. El vacío propio del sujeto que elude su propia realidad y pretende realizarse fuera de ella" (1978a, p. 20). La toma de conciencia ha sido una de las preocupaciones claves del pensamiento latinoamericano contemporáneo, esa

conciencia de ser o estar con los otros será lo que origine tal preocupación. Es en la convivencia donde los hombres toman conciencia, no sólo de su propia existencia, sino también de la existencia de los otros, sus semejantes. Pero una conciencia que no siempre muestra la 'semejanza', el 'ser igual que otros', sino también la diversidad, que puede llegar hasta la negación plena de esa semejanza (1978b, p. 25).

En 1976 sostiene que el filósofo tiene una tarea de ajuste, que consiste en estimular las condiciones que permitan la universalidad de los valores que originó la cultura occidental, ese humanismo que ahora trasciende las fronteras en que se originó y es reclamado universalmente (1976a, p. 46). Frente a la razón de Estado y la respuesta terrorista, cosa que es una aberración, los hombres de razón, los intelectuales han de luchar por hacer prevalecer la única posible: la propia del hombre (siempre en Leopoldo Zea, hombre = ser humano). La del hombre concreto; la razón capaz de comprender y hacerse comprender y a través de esta comprensión hacer patente la igualdad que entre sí guardan todos los hombres de la tierra sin discriminación alguna. Igualdad en la ineludible desigualdad de los hombres entre sí como individuos concretos que son. Ineludible diversidad que al ser comprendida y respetada puede posibilitar la auténtica paz que ha de prevalecer entre los hombres (1993, p. 236).

En síntesis puede decirse que la agenda de Leopoldo Zea para la filosofía consiste en contribuir a la toma de conciencia como afirmación de identidad, lo que debería significar la asunción de la realidad actual y pretérita en vez de estar de espaldas negándola, ello debería permitir la superación de esa constante que ha sido el arar en el mar. Por otra parte, todo ello debe permitir que al interior de ALC y sobre todo en el mundo se acepte la humanidad de los demás en sus diferencias.

\section{UNA AGENDA PARA LA CREACIÓN DE REDES DE INTELECTUALES DE Y SOBRE ALC}

La tarea de organización de redes ha ido constituyendo una sociedad civil del conocimiento en ALC (ver Devés, 2007b), Leopoldo Zea ha sido importante en la articulación entre éstas en el ámbito disciplinario de los estudios sobre ALC, realizándola especialmente a partir del Centro Coordinador y Difusor de Estudios Latinoamericanos (CCYDEL) de la Universidad Nacional Autónoma de México (UNAM). Pero antes de alcanzar esta cristalización, a fines de la década de 1970, tenía ya un largo recorrido en la creación y trabajo con redes internacionales, que se remonta a mediados los años cuarenta del pasado siglo. Es en el marco del CCYDEL donde Leopoldo Zea desarrolla con más fuerza una teorización sobre el papel de 
estas redes respecto de ALC y en la relación entre ALC y el mundo, si puede decirse así. Más aún, puede decirse que el CCYDEL es parcialmente producto de esa misma teorización sobre las redes de estudios de ALC. Recapitulando su trabajo, escribe:

\begin{abstract}
Se venía hablando de la necesidad de coordinar y difundir los trabajos que se hacen en varias de estas instituciones sobre la realidad e identidad común latinoamericana, como punto de partida para posibilitar la buscada integración de la región. Esta preocupación encontró amplio interés en la UNESCO, la cual patrocinó una reunión de expertos en 1976. De esta reunión surgieron recomendaciones que se convertirían en el eje del estímulo que se viene dando a estos estudios en América Latina y en diversas partes del mundo interesadas por los mismos (1990?, p. 1).
\end{abstract}

La tarea de coordinar las distintas instituciones e iniciativas que se ocupan de ALC. De hecho, el CCYDEL se fundó bajo los siguientes temas:

Considerando que la vocación latinoamericanista de la UNAM se fortalecerá coordinando su labor con las que vienen realizando, en el mismo campo, múltiples instituciones internacionales, varias de las cuales han acordado federarse; Considerando que el apoyo que a dichas instituciones ha solicitado redundaría en beneficio de tales estudios; considerando que la UNAM se beneficiaría otorgando el apoyo solicitado, al enriquecer en su seno las posibilidades de intercambio de experiencias, coordinación y difusión de las mismas (citado en Leopoldo Zea 1990?, p. 3).

Por cierto, no bastaba con la fundación, fue necesario mantener y acrecentar las actividades, para lo cual se fueron creando otros mecanismos como la Carta de CCYDEL $L_{L}$ el órgano informativo del CCYDEL (luego CIALC) para los miembros de la SOLAR y la FIEALC. Ésta, señaló Leopoldo Zea, nos permitirá mantenerlos al tanto de las actividades que se llevan a cabo en México y de aquellas que realicen los centros asociados a nuestros dos organismos internacionales, para lo que pedimos su colaboración mediante el aviso oportuno de los eventos (Carta, año 2, $\mathrm{n}^{\circ} 1$ oct-dic. 1984, p. 1).

La creación de redes y de instancias de investigación que deben relacionarse y coordinarse, es comprendida como una parte de la tarea de toma de conciencia de ALC y por ello de afirmación de su identidad. Recuerda Leopoldo Zea que en la citada reunión se consideró "que la búsqueda de la identidad latinoamericana fuese vista como un instrumento de la anhelada integración de la región". Era menester un nuevo esfuerzo en la búsqueda de una integración que había sido intentada política y económicamente. Había que posibilitarlo a través de la toma de conciencia de los latinoamericanos, a través de la educación y la cultura. Se consideró que sería a partir del reconocimiento de la ineludible situación de dependencia colonial que se plantearía la necesidad de su cambio, mediante la promoción de una relación horizontal de solidaridad que hiciese posible la integración. Se consideró igualmente que la toma de conciencia de una identidad común, a través de la educación y la cultura, permitiría lo que por otras vías se había intentado. Ahora bien, para ello era necesario estimular la creación de instituciones que en América Latina se encargasen de hacer patente este conocimiento y ofreciesen los materiales para hacer de ellos instrumentos educativos y culturales para la región. Para el mejor 
cumplimiento de estas recomendaciones, se propuso igualmente, la creación de un Instituto Coordinador de Estudios Latinoamericanos (1990?, p. 1-2). La UNAM, atendiendo a las recomendaciones de la UNESCO y a la especial mención que sobre su expresa preocupación latinoamericanista, decidió patrocinar junto con la Unión de Universidades de América Latina (UDUAL) un simposio que sería el primero para la Coordinación y Difusión de los Estudios Latinoamericanos (1990?, p. 2).

La creación de redes y la coordinación de las diferentes instancias que se ocupan de los estudios latinoamericanos debian afirmar la conciencia, la conciencia debía afirmar la identidad y la identidad debia hacerse presente en el mundo, donde ALC presentara su palabra.

Después del Informe rendido hace 4 años en 1989, el CCYDEL ha continuado y acrecentado sus actividades y su presencia en México, Latinoamérica, Estados Unidos, Europa en su conjunto, Rusia, Asia y África. El CCYDEL ha tenido una presencia activa sobre América Latina, exponiendo el punto de vista latinoamericano sobre los problemas de la región y su relación con los de otras regiones de la tierra (Leopoldo Zea, 1994, p. 8).

\section{UNA AGENDA PARA LA DIFUSIÓN DE LA CULTURA MEXICANA Y DE ALC}

Las dimensiones del pensamiento de Leopoldo Zea que se ponen en relieve y que se intenta desglosar no aparecen por separado en su obra, sino que van articulándose circularmente, remitiéndose unas a otras y también con otras que aquí no han sido consideradas. El quehacer filosófico y la creación de redes se asocian con el quehacer de Leopoldo Zea como agente cultural del Estado mexicano, que concibe y practica como una relación con el mundo, claramente no sólo en México ad intra sino también, e incluso prioritariamente, ad extra. Tzvi Medin señala que "esta postura solidaria con los pueblos dependientes y colonizados se verá reforzada cuando a partir de 1960, Leopoldo Zea se desempeñe como director de la Dirección de Relaciones Culturales de la Secretaría de Relaciones Exteriores, y como tal se encargará precisamente del intercambio cultural". Llevará a cabo viajes por Asia y África y los mismos fortalecerán aún más su postura. Leopoldo Zea se ve sumamente impresionado por el auge del nacionalismo antiimperialista de los pueblos de Asia y África, viendo al nacionalismo como la esencia de esa marea anticolonialista, cualquiera sea su ideología y respetando las diferentes perspectivas posibles: 'El nacionalismo trasformado en socialismo en China, y el nacionalismo que se hace patente en diversas formas en otros pueblos del llamado extremo oriente' (Novedades 9 de enero de 1962, citado Medin 1992, pp. 83-84). Más allá de las divergencias ideológicas, Leopoldo Zea exalta el común denominador de un nacionalismo que implica la reafirmación de la independencia y la soberanía, o sea de esa libertad que constituye para él lo esencial de lo humano. En ese intento de reivindicación nacional de los pueblos oprimidos cree poder detectar y reconocer el viejo ideal cristiano occidental de fraternidad universal (Medin 1992, pp. 83-84).

La afirmación y la proyección de la cultura mexicana, en algún sentido, pasa por la afirmación y proyección de otras voces culturales que si por una parte, suplantarán el discurso monocorde del centro, por otra parte producirán cierta armonía de lo humano. 


\section{ALGUNAS INTERPRETACIONES DE LA AGENDA DE ZEA}

Numerosas personas, de manera más o menos explícita, se han referido a las agendas formuladas por Leopoldo Zea para quienes trabajan con el conocimiento. Se destacan a continuación una serie de autores de importancia que han pensado a partir de Leopoldo Zea y que han formulado (o reformulado) su agenda, varias de los cuales han sido discípulos del propio autor.

Abelardo Villegas, articulando, a la vez que, distinguiendo los estudios eidéticos del quehacer filosófico, destaca que:

la obra de Leopoldo Zea no pretende ser una historia de las ideas, ni una historia a secas. Pretende ser y es una filosofía de la historia de América tal como lo dice el título de uno de sus mejores libros. Siguiendo una idea hegeliana sostiene que la filosofía de la historia pretende crear una conciencia del pasado, entendiendo por ello no el mero hecho de ser consciente, sino un procedimiento intelectual para superar el pasado $(1993,219)$.

Enrique Dussel reinterpretando a Leopoldo Zea, sobre su manera de concebir el filosofar y oponiéndolo al filosofar "hegemónico", formula tres desafíos: "Hay que 1) pensar auto-conscientemente 'desde AL' (con autoconciencia del lugar en la historia mundial), 2) pensar filosóficamente`sobre nuestra realidad' (desde nuestra positividad pero igualmente como dominados, empobrecidos, etc.), y 3) pensar de una manera tal que podamos 'entrar' en la discusión con esa 'comunidad filosófica hegemónica'. Como 'excluidos de ella' debemos 'interpelarla' para que nuestro discurso filosófico propio sea 'reconocido'" (1993, p. 214).

Fernando Aínsa, siguiendo similar línea de reflexión, apunta que la obra de Leopoldo Zea busca crear una conciencia americana y que tener conciencia es saber-en-común y que por tanto ello tiene que ver con la convivencia. Por otra parte, la filosofía americana de Leopoldo Zea no puede ser otra que aquella capaz de resolver el problema de los destinos americanos (1993b, p. 299). La filosofía como una manera de aportar a la solución de los grandes problemas de la dependencia y la identidad de nuestros pueblos (1993a, p. 24).

Pablo Guadarrama González, en parte continuando la interpretación de Villegas y Aínsa, se refiere a la desigual batalla contra los prejuicios que han existido en todos los tiempos, afirmando que, entre quienes poseen la valentía suficiente para emprender una labor emancipatoria, desempeñará un papel primordial la labor de toma de conciencia y que la palabra conciencia es clave en el pensamiento de Leopoldo Zea del mismo modo que lo fue en la obra de Hegel y Marx (1993, pp. 271-272).

Tzvi Medin plantea que Leopoldo Zea concibe la filosofía en México como parte de una confrontación con la situación de dependencia y del constante intento de emancipación, y que de este modo la historia de la filosofía tiene como línea esencial la lucha por la independencia y por la libertad (1992, pp. 33-34). En esta tarea lo mexicano, la autognosis del mexicano y la conformación de la conciencia del mexicano es algo clave (1992, p. 42). Esta labor filosófica de Leopoldo Zea, es 
entendida por Medin, en conexión con la identificación del propio Leopoldo Zea con los nacionalismos anticoloniales que luchan en diferentes partes del mundo por su independencia, cosa que es consecuencia de su humanismo esencial. Es precisamente por este humanismo esencial, piensa Medin que "Leopoldo Zea no tiene problemas (a diferencia de los intelectuales que se identifican con USA o la URSS) en identificarse con los pueblos agredidos por exigir su independencia, sea cual fuere la identidad del agresor" (1992, pp. 82-83).

Javier Pinedo asocia también el tema de los estudios eidéticos con la cuestión de la identidad. Refiriéndose a Leopoldo Zea, a la vez que a Arturo Roig y Arturo Ardao, postula que:

la historia de las ideas, más que un paradigma teórico, se constituye en una declaración de intenciones y principios que intentan abarcar ciertos temas para aproximarse con nuevas energías a la historia, la cultura y la política del continente; un programa que busca entusiasmar y promover el estudio de una determinada realidad, la latinoamericana (1999, p. 29).

Carolina Guerrero, por su parte, señala que uno de los planteamientos que ha signado el compromiso de Leopoldo Zea,

con su trabajo y la proyección del mismo en las sociedades latinoamericanas, ha sido la insistencia sobre la necesidad de elaborar una filosofía política propia, latinoamericana, que soporte y motorice las posibilidades para la emancipación mental y que habría de suceder a la emancipación política de nuestras repúblicas (2003, p. 69).

Ocupándose de Simón Bolívar, Leopoldo Zea:

plantea el dilema del centro y la periferia: los héroes citados por Hegel (Alejandro Magno, Julio César y Napoleón Bonaparte) son expresiones del imperialismo europeo, cuya expansión se despliega hacia los pueblos de la periferia, los cuales toman conciencia de su humanidad y de las posibilidades de la libertad, siendo Bolívar la expresión de esa conciencia periférica. Sostiene Carolina Guerrero que, a partir de ese punto, para Leopoldo Zea "la periferia se convierte en el centro de una libertad que encarna una teleología universalista, dado que en la concepción de Bolívar esa libertad es necesaria a todos los pueblos de la humanidad. Es una reversión substancial del punto de referencia a partir del cual se conoce y se interpreta la historia (2003, p. 69).

Alberto Saladino acude a estas mismas ideas, pero otorgándoles un sentido más amplio y destacando lo que él denomina como "proyecto bolivariano" en la obra de Leopoldo Zea. Saladino apunta a mostrar cómo ese bolivarismo destaca el desenvolvimiento de la semilla producida por el Libertador, la que además renacería una y otra vez, pues muchos de los más importantes autores del pensamiento latinoamericano conciben, a lo largo del tiempo, la necesidad de la integración como condición de la libertad (s/f, p. 3).

Werner Altmann, ocupándose ya no de la filosofía sino de los estudios latinoamericanos y de la creación de redes, destaca el 
incansable papel de misionero del proyecto latinoamericano que Leopoldo Zea ha ejercido por décadas, estimulando en todos los lugares, la formación de centros de estudios latinoamericanos. Señala Altmann que fue esta realidad la que llevó a Roberto Fernández Retamar a calificar a Zea de "Incitator Americae" (2003, p. 7).

\section{CONCLUSIONES Y PROYECCIONES}

Se ha planteado la pregunta por el significado de la noción "pensar más allá del estado-nación en Leopoldo Zea". Ahora bien, ¿cuál es la respuesta entregada por él y por las personas que han reinterpretado su quehacer? o ¿en qué sentido piensa más allá del estado-nación y cuál es la agenda que plantea para ir más allá del estado-nación?

Una primera síntesis sería la siguiente: ALC ha arado en el mar. Para solucionar este problema la propuesta bolivariana de la integración regional sigue siendo válida. Ésta, sin embargo, sólo es posible si se monta sobre una asunción de la realidad, que es conciencia o identidad, lo que permitirá a ALC incidir en el mundo.

Por cierto, sería abusivo pretender proyectar al conjunto de quienes trabajan en ALC con el conocimiento (ese grupo creciente de investigadoras, docentes, funcionarias de la cultura, teóricas de escuelas de pensamiento, ideólogas de partidos, iglesias y otros organismos, etc.) aquello que Leopoldo Zea propuso para los agentes filosóficos o para sí mismo. En este sentido, las propuestas de Leopoldo Zea son inspiraciones no aplicables de modo inmediato a lo que se denomina como intelectualidad. Tampoco pretendió Leopoldo Zea que lo que estaba proponiendo fuera válido para toda la intelectualidad. En todo caso, algunos de los autores revisados más arriba intentan ampliar la agenda propuesta por Leopoldo Zea, haciéndola válida para el conjunto de la comunidad intelectual. Cuestiones como: -la proyección de nuestro quehacer intelectual más allá de la región; -la necesidad de pensar el quehacer intelectual en términos regionales o continentales más que nacionales; -la necesidad de desarrollar una conciencia latinoamericana y del Caribe; y todavía -la posibilidad de elaborar una filosofía de la historia de la región, que tiene que ver con la puesta en relieve de problemas y desafíos que afectan a nuestros pueblos como conjunto; así como -la afirmación de tareas que son igualmente comunes, particularmente la tarea de la integración regional que no es únicamente común sino que en común; -la identificación con los pueblos agredidos, en la exigencia de su independencia, sea cual fuere la identidad del agresor, incluso, por cierto, no limitándose a ALC; -la necesidad de incentivar los estudios sobre la región como conjunto, uno de cuyos ejemplos ha sido el estímulo, en todas parte, a la formación de centros de estudios latinoamericanos; son algunas de que se proyectan a partir de las ideas de Leopoldo Zea.

\section{DIFUSIÓN DE LA CULTURA DE MÉXICO Y DE ALC EN EL MUNDO}

Se ha señalado, que para Leopoldo Zea la afirmación y la proyección de la cultura mexicana, pasa también por la afirmación y proyección de otras voces culturales que, por una parte, suplantarán el discurso monocorde del centro y, por otra, producirán cierta armonía de lo humano. En relación a ello, debe insistirse en que la difusión de la cultura tuvo para Leopoldo Zea casi siempre un sentido vasconceliano: constituirse como un 
aporte desde ALC al mundo, al "mestizaje" universal y síntesis de culturas. Presencia y aporte al mundo, derecho a ser en el mundo mostrando, en pie de igualdad, las diferencias o especificidades culturales. Por otra parte, la acción cultural es concebida como una acción en favor de la libertad de los pueblos colonizados o dependientes. Esta postura solidaria con los pueblos dependientes y colonizados se verá reforzada cuando a partir de 1960 Leopoldo Zea se desempeñe como director de la Dirección de Relaciones Culturales de la Secretaría de Relaciones Exteriores, y como tal se encargue precisamente del intercambio cultural. Los viajes que llevó a cabo por Asia y África fortalecieron aún más su postura.

\section{Estudios eidéticos}

Los estudios eidéticos superando lo nacional y mostrando lo común de las ideas de la región, deben contribuir a la conciencia regional y, por ese camino, a la liberación de la propia región. Los estudios sobre las ideas le han servido para entender que los estados-nación son partes de un conjunto mucho mayor, que es ALC. Las experiencias nacionales se repiten o se reiteran señalando, por esta vía, su propia superación en o hacia un todo mayor que no es otro estado-nación.

Filosofía o pensamiento de ALC

Se ha puesto en relieve que la agenda de Leopoldo Zea para la filosofía consiste en contribuir a la toma de conciencia como afirmación de identidad, lo que debería significar la asunción de la realidad actual y pretérita, para no permanecer de espaldas, negándola. Ello debería permitir la superación de esa constante que ha sido el arar en el mar. Por otra parte, todo ello debe permitir que al interior de ALC y sobre todo en el mundo se acepte la humanidad de los demás en sus diferencias. Ahora bien, la agenda sobre este asunto se traslapa con la anterior sin ser idéntica. Las labores de Leopoldo Zea como estudioso de las ideas y como pensador van (casi) siempre de la mano, aunque se pueden distinguir. El último gran objetivo del pensamiento de Leopoldo Zea más allá del estado-nación fue pensar la globalización y ALC y particularmente México en ese escenario. Pensar la correcta inserción, o la más justa y conveniente, o aquella donde ALC hiciera sus respectivos aportes, pueden ser diversas maneras de formular la propuesta de Leopoldo Zea.

\section{Coordinación de redes}

Si las redes de estudios sobre ALC se explicaban o justificaban en la medida que permitían una coordinación que debía afirmar la conciencia, la conciencia debía afirmar la identidad y la identidad debia hacerse presente en el mundo, donde ALC presentara su palabra, entonces éstas deben entenderse, junto al trabajo en torno al pensamiento, los estudios eidéticos y la acción cultural, como dimensiones de una agenda conjunta, donde los elementos se articulan, y se sinergizan, unos con otros. Estos elementos, se ha planteado, constituyen las maneras relevantes en que Leopoldo Zea apuntó a pensar más allá del estado-nación. 
Pensar más allá del estado-nación

En cada lugar del mundo, escribe Leopoldo Zea, se juega algo de lo que anhelamos, algo de lo que esperamos, algo en lo que creemos. En alguna forma los sucesos en el Mesoriente, en la Europa Central, en el Oriente o en la América del Sur son sucesos nuestros, tienen algo que ver con nosotros. Parece como si nuestro ser se hubiese alargado, prolongado... Nos dolió España en 1936; nos dolió el mundo desgarrado entre 1939-1945; ahora nos ha dolido Guatemala, Marruecos y Argelia y nos duele Egipto y Hungría" (Novedades, 11 de dic, 1956, citado por Medin 1992, p. 83).

Esta solidaridad básica con sucesos ocurridos en diversas partes del mundo que comprometen la libertad y la justicia, es en cierta forma una cuestión de mentalidad y sensibilidad, que permite pensar más allá de la propia aldea. Dicho de otra manera, para esto se requiere una cierta grandeza de miras y de corazón, que impida empantanarse en lo inmediato, del tiempo y del espacio, que impida mirar la realidad con mezquindad o con las anteojeras del aldeano. La mirada de Leopoldo Zea, incluso en su actividad como agente cultural del estado mexicano, pretende abarcar una realidad que lo trasciende o que lo ubica y lo articula a otros estados-nación y a otros pueblos, terminando por ubicarlo en una perspectiva mundial.

Por cierto, las maneras de trascender el estado-nación asumidas por Leopoldo Zea no son las únicas imaginables. Existen otras. Pero éstas le permitieron, por ejemplo, potenciar los estudios latinoamericanos en ALC y más allá, como no ha habido otra persona en la historia de nuestra región. Esto mismo le permitió proyectar la cultura de la región hacia el mundo. Por cierto, dicha labor sólo era posible dadas ciertas condiciones de México: culturales, políticas, estabilidad, diplomacia, etc. Pero la finalidad de este texto no es exaltar la persona de Leopoldo Zea, que sin duda por muchos aspectos lo merece, sino descubrir cómo hizo posible, conceptualmente, iniciativas que exigían una manera específica de concebir las cosas y que, en todo caso, la mayoría de la gente, de México u otros lugares, no fue capaz de realizar. Sin duda las ideas, elaboradas por Leopoldo Zea durante varias décadas, son insumos relevantes para pensar en la actualidad, frente al Bicentenario, cuáles son los criterios más pertinentes para evaluar la trayectoria de ALC y para proyectar la región hacia el futuro.

\section{BIBLIOGRAFÍA}

Aínsa, Fernando (1993a), "Entrevista con L. Zea" en Zea, Leopoldo (compilador) Filosofar a la altura el hombre. Discrepara para comprender, UNAM, México.

Aínsa, Fernando (1993b), "El maestro L. Zea" en ZEA, Leopoldo Filosofar a la altura el hombre. Discrepara para comprender, UNAM, México.

Altmann, Werner (2003), "O latino-americanismo universal de Leopoldo Zea" en Saladino García, Alberto y Santana, Adalberto (Compiladores). Visión de América Latina. Homenaje a Leopoldo Zea, Instituto Panamericano de Geografía y Cultura, Fondo de Cultura Económica, México. 
Devés-Valdés, Eduardo (2007a), Cartas a la intelectualidad, estudiantes universitarias y profesionales del conocimiento en América Latina y el Caribe, Grafitti, Santiago.

Devés-Valdés, Eduardo (2007b), Redes intelectuales en América Latina, Hacia la constitución de una comunidad intelectual, Instituto de Estudios Avanzados, Universidad de Santiago de Chile, Santiago.

Devés-Valdés, Eduardo (2003), "Leopoldo Zea más allá de sus 90 años" en Saladino García, Alberto y Santana, Adalberto (Compiladores) Visión de América Latina. Homenaje a Leopoldo Zea, Instituto Panamericano de Geografía y Cultura, Fondo de Cultura Económica, México.

Devés-Valdés, Eduardo (2000, 2003, 2004), El Pensamiento Latinoamericano en el Siglo XX. Entre la modernización y la identidad. Tomo I, Del Ariel de Rodó a la CEPAL (1900-1950), Tomo II Desde la CEPAL al neoliberalismo (1950-1990), Tomo III. Las discusiones y las figuras del fin de siglo. Los años 90 (1990-2000), B Biblos-DIBAM, Santiago-Buenos Aires.

Devés-Valdés, Eduardo y Melgar-Bao, Ricardo (2005), “El pensamiento de Asia en América Latina. Hacia una cartografía", en Revista de Hispanismo Filosófico, $\mathrm{N}^{\circ}$ 10, Fondo de Cultura Económica, Madrid.

Dussel, Enrique (1993), "El proyecto de una filosofía de la historia latinoamericana" en ZEA, Leopoldo (Compilador) Filosofar a la altura del hombre. Discrepar para comprender, UNAM, México.

Górski, Eugeniusz (1994), Dependencia y originalidad de la filosofía en Latinoamérica y en la Europa del Este, UNAM, México.

Guadarrama González, Pablo (1993) “Urdimbres del pensamiento de L. Zea frente a la marginación y la barbarie" en ZEA, Leopoldo Filosofar a la altura el hombre. Discrepara para comprender, UNAM, México.

Guerrero, Carolina (2003), “Leopoldo Zea y una perspectiva original sobre Bolivar como hombre político" en Saladino García, Alberto y Santana, Adalberto (Compiladores) Visión de América Latina. Homenaje a Leopoldo Zea, Instituto Panamericano de Geografía y Cultura, Fondo de Cultura Económica, México.

Medin, Tzvi (1992), Leopoldo Zea: ideología y filosofía de América Latina, UNAM, México.

Palti, Elías (2003), "L. Zea y la historiografía de las ideas en América Latina", en Saladino García, Alberto y Santana, Adalberto (Compiladores) Visión de América Latina. Homenaje a Leopoldo Zea, Instituto Panamericano de Geografía y Cultura, Fondo de Cultura Económica, México.

Pinedo, Javier (1999), “Identidad y método: aproximaciones a la historia de las ideas en América Latina" en Cancino Troncoso, Hugo, KELGEL, Susanne y Leonzo, 
Nancy (eds) Nuevas perspectivas teóricas y metodológicas de a historia intelectual en América Latina, Iberoamericana, Verbuert.

Saladino, Alberto (s/f) "El proyecto bolivariano en el pensamiento de Leopoldo Zea", spi.

Villegas, Abelardo (1993), “La filosofía como compromiso" en Zea, Leopoldo (Compilador) Filosofar a la altura del hombre. Discrepar para comprender, UNAM, México.

Villoro, Luis (1993), "Sobre el problema de la filosofía latinoamericana" en Zea, Leopoldo (Compilador) Filosofar a la altura del hombre. Discrepar para comprender, UNAM, México.

Wiredu, Kwasi (1995), Conceptual decolonization in philosophy, Hope Publications, Ibadan.

www.internacionaldelconocimiento.org

Zea, Leopoldo (1999), Geopolítica de América Latina y el Caribe, "Presentación", compilado por Leopoldo Zea y Mario Magallón Anaya.

Zea, Leopoldo (1994) Informe de actividades del Centro Coordinador y Difusor de Estudios Latinoamericanos 1989-1994, UNAM, México.

Zea, Leopoldo (1990?), Diez años del Centro Coordinador y Difusor de Estudios Latinoamericanos 1979-1989, UNAM, México.

Zea, Leopoldo (1984), Carta CCYDEL, año 2, n ${ }^{\circ} 1$ oct-dic 1984, UNAM, México.

Zea, Leopoldo (1982), “Prólogo” a RIZAL, José Noli me tangere, Biblioteca de Ayacucho, Caracas.

Zea, Leopoldo (1978a), Filosofía de la historia americana, Fondo de Cultura Económica, México.

Zea, Leopoldo (1978b), Conciencia y posibilidad del mexicano, Porrúa, México.

Zea, Leopoldo (1976a), El pensamiento Latinoamericano, Ariel, Barcelona.

Zea, Leopoldo (1976b), Dialéctica de la conciencia americana, Alianza Editorial, México.

Zea, Leopoldo (1959), La cultura y el hombre de nuestros días, UNAM, México. 